\title{
Zur semantischen Basis der Numerusdistinktion im gesprochenen Portugiesisch Brasiliens
}

\author{
Enrique Huelva Unternbäumen (Brasilia)
}

\begin{abstract}
This paper examines the semantics of the singular/plural distinction in spoken Brazilian Portuguese. Analysis of real data from this variety of Portuguese shows that: 1) both plural marked NPs and plural unmarked NPs may have a plural reference; 2) the difference in plural marking is determined by semantic differences pertaining to the properties of (types of) plural objects denoted, respectively, by one or other construction.

KEYWORDS: Semantics; Number; Brazilian Portuguese.
\end{abstract}

\section{$1 \quad$ Einleitung}

Die linguistische Beschäftigung mit dem gesprochenen brasilianischen Portugiesisch (im Folgenden GBP) hat in den letzten Jahren eine Reihe von Untersuchungen hervorgebracht, die in besonderer Weise auf die Beschreibung grammatikalischer Merkmale dieser Varietät des Portugiesischen fokussieren. Diesen Untersuchungen ist es teilweise gelungen zu zeigen, dass sich das GBP in Bezug auf zentrale grammatikalische Phänomene vom "Standardportugiesisch" bzw. vom gesprochenen Portugiesischen Portugals stark unterscheidet - sogar derart, dass die Frage als berechtigt erscheint, ob die ans Licht gebrachten grammatikalischen Unterschiede nicht als Indizien dafür interpretierbar sind, dass das GBP in Bezug auf die beobachteten Phänomene im Begriff ist, sich sprachtypologisch von anderen Varietäten des Portugiesischen bzw. vom "Standardportugiesisch" zu entfernen. ${ }^{1}$

Unter anderem sind dabei wesentliche Unterschiede hinsichtlich der Realisierung der grammatikalischen Kategorie Numerus innerhalb von NPs konstatiert worden. Dabei haben sich allerdings bisherige Arbeiten beinahe ausschließlich auf die Beschreibung und Erklärung von Fällen wie den folgenden beschränkt:

$(1)^{2}$

(a) $\quad$...eu

estou calculando

essas

carreta ...

ich bin

berechnen-Gerund.

diese-pl.

LKW-Ladung

'ich bin dabei, die LWK-Ladungen zu berechnen'

\footnotetext{
${ }^{1}$ Vgl. dazu etwa die Aufsätze in Roberts/Kato (eds.) (1996) und in Große/Zimmermann (eds.) (1998).

2 Alle Beispiele stammen aus dem Corpus Gesprochenes Portugiesisch Brasiliens der Fakultät für Linguistik und Literaturwissenschaft der Universität Bielefeld.
} 


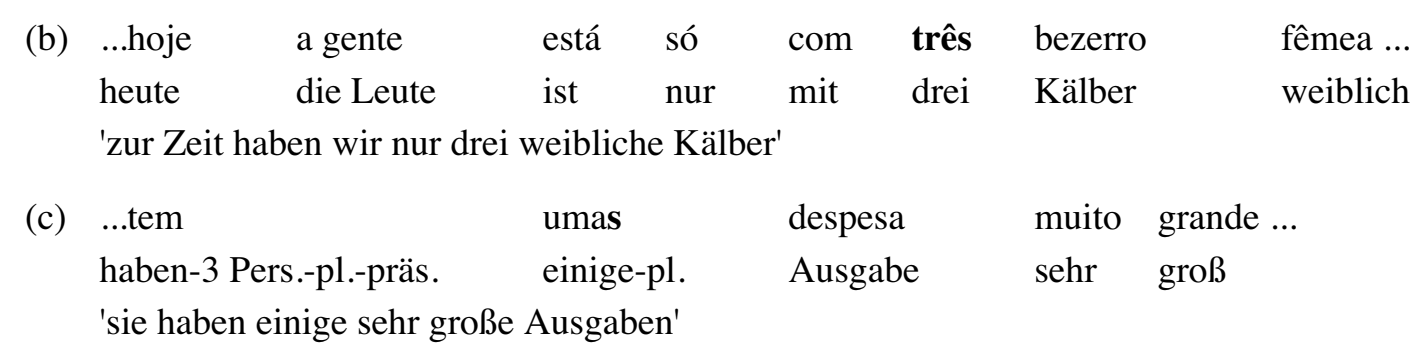

Offensichtlich liegt hier ein Abbau des nominalen Kongruenzsystems als Verfahren zur Markierung von grammatikalischen Dependenzrelationen zwischen den einzelnen konstitutiven Bestandteilen von komplexen NPs vor. Die Kategorie Numerus wird meistens nur an der ersten Position - morphologisch durch den Pluralmarker $-\boldsymbol{s}^{3}$ (vgl. (1)(a) und (c)) oder lexikalisch (vgl. (1)(b)) - markiert. ${ }^{4}$ Dagegen ist die Markierung der Kategorie Numerus im Standardportugiesisch nicht selektiv, sondern distributiv. Numerus wird am Determinierer, am Quantifizierer, am adjektivischen Attribut und am nominalen Nukleus selber markiert: ${ }^{5}$

(2) ...Aqueles

poucos vestidos

vermelhos ...

(Det.

Quant.

Nom.

Adj.)

diese-pl.

wenigen

Kleider

rot-pl.

'diese wenigen roten Kleider'

Ein hiervon - trotz einer gewissen oberflächlichen Ähnlichkeit - zu unterscheidendes und in der Literatur bislang kaum beachtetes Phänomen ${ }^{6}$ kann durch den Gebrauch von NPs in Äußerungen wie in (3) veranschaulicht werden, in denen im Unterschied zum Standardportugiesisch, aber auch entgegen den Erwartungen von Sprechern etwa des Spanischen oder des Deutschen, kein Element der jeweiligen NP sowie kein mit der NP kongruierendes externes Element (vgl. (3)(b)) eine Pluralmarkierung aufweist.
(a) ...Luana,
você já
escovou o
Luana du schon putzen-2Pers.-sg.-prät. der
dente? ...
'Luana, hast du dir schon die Zähne geputzt?'
(b) ...quanto custa a laranja?...
wieviel kostet die-sg. Orange
'wieviel kosten die Orangen?'
(c) ...Vai
lavar gehen-2Pers.-sg.-imper. waschen
a mão! ...
'geh mal die Hände waschen!'

\footnotetext{
${ }^{3} \mathrm{Zu}$ besonderen Regeln für die Bildung des Plurals vgl. etwa Cunha/Cintra (2001: 181ff.).

4 Vgl. dazu etwa Naro/Pereira Scherre (2000), Vazzata-Dias (2000) und aus der deutschsprachigen Lusitanistik Gärtner (1996). Zu der Entwicklung von distributiven zu selektiven Formen der Markierung innerhalb der NP vgl. im Allgemeinen Ole (2001: 1626-1627) und Ronneberger-Sibold (1997) für das Deutsche.

5 Genauer: Nukleus und Modifikatoren des Nukleus, die nicht durch eine Präposition eingeleitet werden.

${ }^{6}$ Eine Ausnahme bildet mit Sicherheit die Arbeit von Farrell (1998), die sich allerdings nicht auf empirisches Material stützt.
} 

im gesprochenen Portugiesisch Brasiliens

(d)

$\begin{array}{lllll}\text {...O meu pai } & \text { sempre deixa } & \text { a } & \text { chuteira } \\ \text { der } & \text { mein } & \text { Vater } & \text { immer lassen-3Pers.-sg.-präs. } & \text { die-sg. Fussballschuh } \\ \text { no } & \text { portamalas ... } & & \\ \text { im } & \text { Kofferraum } \\ \text { 'mein Vater lässt immer die Fussballschuhe im Kofferraum' }\end{array}$

Trotz fehlender morphosyntaktischer oder lexikalischer Pluralmarkierung haben alle in (3) fett markierten NPs in der jeweiligen Verwendungssituation (nicht-generische, partikuläre) Pluralreferenz. Etwa in (3)(a) fragt der Sprecher die Hörerin, ob sie sich alle Zähne - und nicht etwa einen einzigen Zahn - geputzt hat. Die nicht-plural-markierte NP o dente referiert auf mehr als eine Entität (alle Zähne der Hörerin). Analog dazu fordert der Sprecher den Hörer mit der Verwendung der NP a mão in (3)(c) selbstverständlich dazu auf, sich nicht nur eine Hand zu waschen, sondern gleich beide. Auch hier haben wir also eine nicht-pluralmarkierte NP, die auf mehr als eine außersprachliche Entität referiert.

Sowohl die fett markierten NPs in (3) als auch jene oben in (1) zeichnen sich formal dadurch aus, dass sie einen Determinierer (DET) aufweisen. ${ }^{7}$ Es handelt sich also nicht um so genannte bloße ('bare') NPs. Etwa in (1)(a) wird der nominale Nukleus carreta durch das Demonstrativum essas determiniert; in (3)(a) wird der nominale Nukleus dente durch den definiten Artikel $o$ determiniert, usw. Zwischen den NPs in (1) und den NPs in (3) besteht jedoch auch ein formaler Unterschied. Wie oben bereits erwähnt, haben die NPs in (1) einen offen realisierten Pluralisierer. Dabei handelt es sich um das pluralisierende Suffix $-\boldsymbol{s}$ (wie in (1)(a) und (c)) oder um lexikalische Elemente, deren Semantik bereits Pluralität signalisiert (Numeralia wie in (1)(b), aber in weiteren Fällen auch andere Quantifizierer und Strukturen wie z.B. um monte de, uma porção de, etc. (ein Haufen, eine Menge)). ${ }^{8}$ Im Gegensatz dazu verfügen die NPs in (3) - wie bereits erwähnt - über keine offen realisierte (morphosyntaktische oder lexikalische) Pluralmarkierung. Die NP o dente in (3)(a) etwa kommt in der neutralen (Numerus unmarkierten, für das Singular üblichen) Form vor. Sie weist auch keine Pluralität markierenden lexikalischen Elemente auf. Ihre Pluralreferenz wird also weder morphologisch noch lexikalisch markiert. Das ist auch der Fall bei $a$ mão in (3)(c) und $a$ chuteira in (3)(d). Bemerkenswert ist auch, dass in (3)(b) das morphosyntaktische Verfahren der (externen) Kongruenz zwischen der Subjetk-NP a laranja und dem Verb custa (3 Person Singular) auch nicht zur Markierung der Pluralreferenz der NP verwendet wird.

Die skizzierten formalen Eigenschaften der NPs in (1) und (3) können schematisch wie folgt dargestellt werden.

(a)

[PL DET N]

(b)

\section{[DET N]}

Abbildung 1

\footnotetext{
${ }^{7}$ Ich verwende den Terminus 'Determinierer' als Oberbegriff für Artikel, Demonstrativa und Quantifizierer.

${ }^{8} \mathrm{Zu}$ dem Spektrum an Möglichkeiten für die offene Signalisierung von Pluralität im GBP vgl. etwa Pereira Scherre (1998).
} 
In Abbildung 1 steht PL für Pluralisierer, DET für Determinierer und $\mathrm{N}$ für Nomen. ${ }^{9}$ In Anlehnung an die Kognitive Grammatik möchte ich davon ausgehen, dass die dargestellten Strukturen zwei unterschiedliche grammatikalische Konstruktionen zur Bildung von NPs im GBP repräsentieren. ${ }^{10}$ Der wesentliche formale Unterschied zwischen ihnen besteht - wie erwähnt - in der Präsenz (Konstruktion (a)) bzw. Absenz (Konstruktion (b)) eines offen realisierten Pluralisierers.

Semantisch können sowohl die fett markierten NPs in (1) als auch jene in (3) vorläufig durch die Beobachtung charakterisiert werden, dass sie Pluralreferenz besitzen. D.h.: sie alle referieren auf mehr als eine Entität. Ferner ist für die NPs in (3) und für alle von mir untersuchten NPs dieses Typs (also NPs mit Pluralreferenz und ohne offen realisierte Pluralisierer) charakteristisch, dass ihr jeweiliger nominaler Nukleus auf physikalisch konkrete "Dinge" im Sinne eines bestimmten Typs von materiellen Entitäten referieren (Jackendoff 1990: 20; vgl. mão, dente, laranja, chuteira in (3) und auch die Beispiele unten in Kapitel 3). Im Gegensatz dazu wurde in unseren Daten festgestellt, dass die NPs mit offen realisiertem Pluralisierer auch auf nicht physikalisch konkrete Dinge (wie z.B. as pergunta (dt.: die Fragen), essas dúvida, (dt.: diese Zweifel) etc. referieren.

Im Folgenden soll gezeigt werden, dass der dargestellte formale Unterschied zwischen beiden Konstruktionen (nämlich Präsenz vs. Absenz eines offen realisierten Pluralisierers) in systematischer Weise mit semantischen Faktoren - oder genauer: mit den semantischen Eigenschaften der jeweils kodierten Pluralobjekte - korreliert. Um dies zu tun, müssen wir zuerst jedoch noch einige zentrale konzeptuelle Aspekte kurz besprechen.

\section{$2 \quad$ Konzeptuelles}

Der zentrale Begriff der grammatikalischen Konstruktion wird hier im Sinne der Kognitiven Grammatik verwendet (vgl. u. a. Langacker 1987; 1991a; 1991b; Croft 2002). ${ }^{11}$ Für die Kognitive Grammatik sind Konstruktionen konventionalisierte symbolische Einheiten (Langacker 1987: 57-63). Einfach dargestellt, sind Konstruktionen kognitiv fixierte Routinen (Einheiten), die in einer bestimmten Sprachgemeinschaft verwendet werden (konventionalisierter Charakter) und die eine feste Verknüpfung von Form (formaler Pol) und Bedeutung (semantischer Pol) umfassen (symbolischer Charakter).

\footnotetext{
${ }^{9}$ Die Abbildung soll lediglich die konstitutiven Bestandteile beider Konstruktionen, nicht jedoch ihre lineare Abfolge darstellen. Denn - wie oben gezeigt - der Pluralisierer wird in der Konstruktion (a) meistens suffixal am Determinierer realisiert.

10 Zum Konzept der grammatikalischen Konstruktion im Allgemeinen vgl. unten Kap. 1. Eine ausführliche Darstellung nominaler Konstruktionen aus der Sicht der Kognitiven Grammatik finden wir bei Langacker (1991: $156 \mathrm{ff}$.).

${ }^{11}$ Kognitive Grammatik soll hier im weitesten Sinne als Bezeichnung für ein theoretisches Paradigma der aktuellen grammatikalischen Theoriebildung verstanden werden, das eine Reihe einzelner, in ihren Grundannahmen übereinstimmenden Theoriemodelle umfasst. Für die folgenden Überlegungen werden wir vor allem auf das Modell von Langacker $(1987 ; 1991)$ und auf die "Radical Construction Grammar" von Croft (2001) rekurrieren.
} 
Lexikon, Morphologie und Syntax sind ausschließlich und vollständig auf der Grundlage von symbolischen Einheiten beschreibbar. Wichtig ist dabei, dass sie ein Kontinuum bilden, das nicht in separate Komponenten getrennt werden kann (vgl. Langacker 1991a: 514; Croft 2002: 17ff.; 2004: 2). Konsequenterweise haben alle Elemente einer Sprache einen formalen und einen semantischen Pol. Das bedeutet, dass auch alle grammatikalischen Konstruktionen Bedeutung besitzen, auch wenn diese in der Regel abstrakter ist, als jene von lexikalischen Einheiten.

Ein weiterer wichtiger Grundsatz der Kognitiven Grammatik ist die Gleichsetzung von Bedeutung und Konzeptualisierung (Langacker 1991a: 515-516; 1991b: 2ff.). Die Bedeutung einer grammatikalischen Konstruktion besteht demnach in einer bestimmten, konventionalisierten Art und Weise, konzeptuelle Inhalte zu "konstruieren". Es ist in diesem "konzeptualistischen" Sinne, dass grammatikalische Konstruktionen "bedeutungsvoll" sind: "grammar (...) is meaningful because it embodies and symbolizes a particular way of construing conceptual content" (Langacker 1991a: 517).

Der semantische Gehalt (semantischer Pol) einer grammatikalischen Konstruktion ist in der Regel hoch abstrakt und schematisch. Er besteht meistens aus einer Reihe von konzeptuellen Kategorien, die eher als Parameter, die mit unterschiedlichen Werten belegt werden können, zu konzeptualisieren sind, und aus einer bestimmten Art der Kombination bzw. der Relationierung zwischen diesen Kategorien. Talmy (2002) unterscheidet z. B. u. a. Kategorien wie plexity, boundedness, dividedness, usw. ${ }^{12}$ Der parameterähnliche Charakter dieser Kategorien manifestiert sich darin, dass sie je nach grammatikalischer Konstruktion mit unterschiedlichen Werten belegt werden können. So kann etwa die Kategorie plexity den Wert uniplex (etwa im Konzept 'AUTO') oder multiplex (wie etwa im Konzept 'AUTOS') aufweisen. ${ }^{13}$ Einzelne konzeptuelle Kategorien können meist aufgrund funktionaler Kriterien zu größeren konzeptuellen Strukturen bzw. Systemen verknüpft werden (vgl. Talmy 2002: 40ff.). ${ }^{14}$ So entsteht etwa aus der Verbindung der Kategorien plexity, boundedness, dividedness das komplexe konzeptuelle System Configurational Structure, das in der Regel für schematische Strukturierungen in Raum und Zeit verwendet wird. Mit diesem System werden wir uns weiter unten umfassender beschäftigen (vgl. Kap. 2).

Grammatikalische Konstruktionen bewirken eine bestimmte Konstruktion konzeptuellen Gehalts insofern, als sie die abstrakten, schematischen Kategorien, die sie kodieren, mit dem semantischen Gehalt lexikalischer Einheiten integrieren. ${ }^{15}$ Aus dieser Integration bzw. Verbindung zwischen dem semantischen Pol lexikalischer Einheiten und dem semantischen Pol grammatikalischer Konstruktionen resultiert die Gesamtbedeutung, die von einer bestimmten komplexen Struktur ausgedrückt wird.

\footnotetext{
${ }^{12}$ Zur Bedeutung dieser Kategorien vgl. unten Kapitel 2.

${ }^{13}$ Großschreibung bedeutet, dass es sich um Konzepte bzw. um außersprachliche Entitäten handelt.

${ }^{14}$ Langacker (1987:147-182 ) verwendet dafür den Terminus domain. Eine umfassendere terminologische und konzeptuelle Auseinandersetzung mit diesem Themenkomplex finden wir bei Clausner/Croft (1999).

${ }^{15} \mathrm{Zu}$ dieser Aufgabenverteilung zwischen lexikalischen und grammatikalischen Einheiten vgl. Talmy (2002: 21$40)$.
} 
In Anlehnung an die Kognitive Grammatik sollen die oben in Abbildung 1 repräsentierten Strukturen als zwei unterschiedliche (nominale) grammatikalische Konstruktionen des GBP betrachtet werden, die sich nicht nur formal, sondern auch semantisch hinsichtlich des von ihnen jeweils kodierten semantischen Pols differenzieren. Die zentrale Aufgabe der vorliegenden Untersuchung liegt demnach darin, die entsprechenden semantischen Pole dieser Konstruktionen zu beschreiben und bestehende Differenzen zwischen ihnen herauszuarbeiten.

Eins der schwierigsten Probleme bei der Charakterisierung des semantischen Pols grammatikalischer Konstruktionen, also bei der Charakterisierung grammatikalisch relevanter konzeptueller Kategorien, liegt darin zu bestimmen, als wie "fein graniert" wir uns diese Kategorien vorstellen sollen. ${ }^{16}$ Der vorliegende Fall bildet dabei keine Ausnahme.

Für Jackendoff kann man diese zentrale Frage am besten klären, wenn man eine Analogie zwischen der Evolution der Physik in den letzten 100 Jahren und der Evolution der Linguistik zieht (vgl. u. a. Jackendoff 1991: 11-13; 2002: 334-339). So stellt er fest, dass einer der bahnbrechensten Momente der Geschichte der Physik die Dekomposition aller Substanzen in 92 primitive Elemente war, dass diese jedoch wiederum weiter dekomponiert wurden, zunächst in Elektrone und Nukleus, dann in Elektrone, Protone und Neutrone, die alle ihrerseits in Quarks, welche auch weiter dekomponierbar sind. Analog dazu sind linguistisch relevante konzeptuelle Kategorien wie etwa DING, GRUPPE, SUBSTANZ, PLURAL etc. als spezifische Kombinationen von den grundlegenderen, fein granierteren Kategorien $[ \pm$ begrenzt] und [ \pm interne Struktur] beschreibbar (Jackendoff 1991: 20ff.).

Diese Analogie trifft jedoch nicht ganz zu. Das Weiter-Dekomponieren in immer kleinere, feiner granierte Einheiten mag für die Physik eine natürliche, gegenstandsadäquate theoretische Entwicklungstendenz und eine richtige Beschreibungsstrategie darstellen, die immer weiter, ohne absehbares logisches Ende getrieben werden kann. In der Linguistik ist es etwas anders. Denn der Möglichkeit des Einsatzes dieses Verfahrens werden durch die eigene Natur linguistischer Fakten gewisse Grenzen gesetzt. Wie oben erwähnt, sind linguistische Einheiten stets symbolischer Natur. Eine bestimmte linguistische Form selegiert eine konzeptuelle Kategorie (bzw. eine spezifische Kombination konzeptueller Kategorien) als ihren semantischen Pol. Analog dazu selegieren gesamte linguistische Subsysteme bestimmte Typen von konzeptuellen Kategorien und weisen andere als durch sie nicht kodierbar ab (vgl. dazu Talmy 2002: 24-40). Die Antwort auf die methodologisch zentrale Frage, ob eine bereits ermittelte, linguistisch kodierte konzeptuelle Kategorie weiter dekomponiert werden soll, hängt demnach davon ab, ob die aus der Zerlegung resultierenden kleineren Bestandteile jeweils als autonome (d. h. vor allem auch als bedeutungsdifferenzierende) Elemente des semantischen Pols linguistischer Einheiten fungieren. Es würde beispielsweise keinen Sinn machen, die Kategorie $[ \pm$ begrenzt $]$ hinsichtlich etwa der möglichen Typen von konzeptualisierbaren Grenzen weiter zu dekomponieren, wenn keine linguistischen Einheiten ermittelt werden könnten, die für diese Differenzierung sensitiv wären (vgl. dazu Kap. 2.). Die einzelnen Subsysteme der Sprache sowie einzelne linguistische Einheiten selegieren nicht nur

\footnotetext{
16 Vor allem Talmy (2002: 18-40) versucht, die Eigenschaften von grammatikalisch relevanten konzeptuellen Kategorien in expliziter Weise zu charakterisieren und mit lexikalisch kodierten Kategorien zu vergleichen.
} 
die konzeptuellen Kategorien, die sie jeweils kodieren, sondern auch, wie fein graniert diese Kategorien sind.

Diese Selektionen weisen außerdem bis zu einem gewissen Grad wichtige einzelsprachliche Besonderheiten auf. Talmy (2002: 38) weist in diesem Zusammenhang zwar auf die Wahrscheinlichkeit der Existenz eines "universally available inventory of grammatically specificable concepts" hin, räumt aber zugleich ein, dass dies nicht heißen muss, dass diese Konzepte auch universal realisiert werden (Talmy 2002: 39). Des Weiteren sieht er es als eine zentrale Aufgabe der Linguistik "to determine whatever principles may govern the nature of the selection from the overall inventory for occurence within a given language" (Talmy 2002: 40). Auch Croft differenziert in seiner Radical Construction Grammar zwischen einem unversalen konzeptuellen Raum (conceptual space) und einzelsprachlichen Strukturierungen dieses Raums (semantic maps) (Croft 2002: 92-98).

Einen dieser Unterschiede zwischen einzelnen Sprachen haben wir in den vorangegangenen Überlegungen bereits angedeutet: Sprachen scheinen sich hinsichtlich der Granularität der konzeptuellen Kategorien zu unterscheiden, die durch funktional ähnliche grammatikalische Konstruktionen kodiert werden. In der Terminologie Jackendoffs könnte man auch sagen, dass es Sprachen gibt, die konzeptuelle Kategorien (bzw. komplexe konzeptuelle Systeme) auf einer tiefereren Dekompositionsebene kodieren als andere. Um diesen Sachverhalt besser zu erfassen, möchte ich hier den Begriff der Granularität der Kodierung einführen. Die Ausdrücke grob- bzw. feingranular werden dann verwendet, um (relative) Unterschiede in der Granularität der Kodierung zwischen funktional ähnlichen Konstruktionen darzustellen.

Differenzen hinsichtlich der Granularität der Kodierung zwischen unterschiedlichen Sprachen (oder zwischen unterschiedlichen Konstruktionen einer einzigen Sprache) sind bislang meines Wissens nocht nicht in systematischer Form untersucht worden. Es gibt jedoch durchaus Studien, die diesen Sachverhalt in Bezug auf einzelne konzeptuelle Systeme konstatiert haben. Hinsichtlich des für die vorliegende Untersuchung wichtigen konzeptuellen Systems der Configurational Structure stellen z. B. Behrens (1995), Behrens/Sasse (1999) und Blühdorn/Simões/Schmalz (2004) fest, dass die konzeptuellen Kategorien zählbar versus nicht-zählbar (bzw. Masse) zwar im Englischen in holistischer Form (also ohne weitere Dekomposition ihrer Bestandteile) kodiert werden, ${ }^{17}$ nicht so jedoch in anderen Sprachen wie etwa dem Ungarischen und dem Arabischen (vgl. Behrens 1995) oder etwa dem Portuguiesischen (Blühdorn/Simões/Schmalz 2004). Vielmehr verfügen diese Sprachen über grammatikalische Konstruktionen, die schon sensitiv für die konzeptuellen Basiskomponenten der Distinktion zählbar versus nicht-zählbar sind. ${ }^{18}$ Wenn dies zutrifft, kann man sagen, dass das Englische bezüglich des konzeptuellen Subsystems der Distinktion zählbar versus nichtzählbar vergleichsmäßig grobgranular ist, während die anderen erwähnten Sprachen eher als feingranular einzustufen sind.

\footnotetext{
${ }^{17}$ Vgl. jedoch u.a. Jackendoff (1991) und Langacker (1991: 73 ff.).

18 Wie etwa [ \pm individualisiert $]$ und $[ \pm$ inkrementiert $]$ ) im Portuguiesischen. Vgl. dazu Blühdorn/Simões/ Schmalz (2004: 10ff.).
} 
Wie oben bereits erwähnt, referieren die NPs, die nach den in Abbildung 1 dargestellten Konstruktionen gebildet werden, auf mehr als ein Verweisobjekt. Die beiden Typen von NPs unterscheiden sich jedoch hinsichtlich der genauen Eigenschaften der Pluralobjekte, die sie jeweils kodieren. D. h.: das GBP hat in der komplementären Verwendung dieser nominalen Konstruktionen - wie unten zu zeigen sein wird - ein grammatikalisches Mittel, das über die einfache Kodierung von Pluralität hinausgeht und für das sprachliche Erfassen von weiteren, feiner granierten Eigenschaften von Pluralobjekten sensitiv ist. Ein zentraler methodologischer Schritt der vorliegenden Untersuchung besteht demnach darin, diese feiner granierten Eigenschaften zu ermitteln.

\section{Das konzeptuelle Subsystem der Multiplexität}

Der Bereich des konzeptuellen Raums, der für die Beschreibung der Unterschiede zwischen den semantischen Polen der oben skizzierten Konstruktionen des GBP relevant ist, ist der der Multiplexität. Multiplexität kann als ein Subsystem des komplexeren kognitiven Systems betrachtet werden, das Talmy (2002: 47-68) Configurational Structure nennt. Nach Talmy (2002: 47) umfasst dieses komplexe System "the schematic structuring or geometric delineations in space or time or other qualitative domain (...)". Für unsere Zwecke sind insbesondere drei schematische konzeptuelle Kategorien dieses komplexen Systems von Interesse: plexity, state of boundedness und degree of dividedness. Zusätlich zu diesen bereits von Talmy genannten Kategorien soll unten die Kategorie Partikularisierung als weiteres konstitutives Element des konzeptuellen Subsystems der Multiplexität eingeführt werden.

Die semantische Kategorie plexity ist umfassender als die traditionelle Kategorie Numerus. Denn sie erfasst nicht nur den Unterschied zwischen einem einzelnen Element und mehreren Elementen (eines Typs) im Bereich des Raums (also materielle Elemente), sondern auch der Zeit (Prozesse, Ereignisse, Zustände, etc.). Diesem intendierten allgemeinen Sinne entsprechend, definiert Talmy diese Kategorie als "a quantity's state of articulation into equivalent parts", wobei die Quantität räumlich oder zeitlich sein kann (Talmy 2004: 48). Die Kategorie plexity kann durch zwei unterschiedliche Werte belegt werden: uniplex (z. B. beim Konzept AUTO) oder multiplex (wie z. B. beim Konzept AUTOS). ${ }^{19}$

Auch die Kategorie state of boundedness (Talmy 2002: 50-55) fasst Ähnlichkeiten zwischen den Bereichen Raum und Zeit zusammen. Und auch sie kann zwei Werte aufweisen: begrenzt, wenn die Konzeptualisierung einer Entität eine intrinsische Referenz auf eine Grenze bzw. Begrenzung umfasst (und folglich die Entität als eine individualisierte Einheit dargestellt wird, z. B beim Konzept SEE), und unbegrenzt, wenn dies nicht der Fall ist (z. B. beim Konzept WASSER).

Die dritte Kategorie, die für uns relevant ist, ist state of dividedness (Talmy 2002: 55-58). Sie bezeichnet die Konzeptualisierung der internen Beschaffenheit bzw. Struktur einer Entität. Eine Entität ist zusammengesetzt (z. B. das Konzept BÄUME) wenn ihre interne Beschaffen-

\footnotetext{
${ }^{19}$ Ich halte den Terminus plexity (und konsequenterweise auch uniplex und multiplex) für etwas verwirrend, denn er bezeichnen bei Talmy (2002) lediglich einen Aspekt der Konzeptualisierung der Multiplexität von Objekten, Handlungen, Tätigkeiten, etc.
} 
heit so konzeptualisiert wird, dass sie Brüche, Unterbrechungen oder Ähnliches aufweist, d. h. wenn sie aus diskreten Elementen besteht. Ansonsten ist sie kontinuierlich (z. B. beim Konzept WASSER).

Neben diesen drei von Talmy beschriebenen Kategorien ist für den Bereich der Multiplexität eine vierte Kategorie relevant, die ich Partikularisierung nennen möchte. Es handelt sich dabei um das Ergebnis eines Prozesses der Hervorhebung, also der Konstruktion einer Figur und eines Hintergrunds in der Konzeptualisierung einer bestimmten Entität. ${ }^{20}$ In einer Entität, deren interne Beschaffenheit als zusammengesetzt konzeptualisiert wird, können einmal die konstitutiven diskreten Elemente hervorgehoben werden. In diesem Fall bilden sie die Figur und der Einheitscharakter der zusammengesetzten Entität den Hintergrund. Im ungekehrten Fall kann der Einheitscharakter selber hervorgehoben werden, wobei dann die konstitutiven diskreten Elemente in den Hintergrund treten. Um beide entgegengesetzten Fälle zu erfassen, möchte ich die Wertebezeichnungen partikularisiert vs. nicht-partikularisiert einführen.

Diese Kategorie ist leicht verwechselbar mit dem state of dividedness. Es handelt sich jedoch um zwei unabhängige Kategorien, auch wenn eine bestimmte logische Kovariation feststeht: eine Möglickeit der Hervorhebung des einen oder des anderen Typs besteht nur, wenn eine Entität als intern zusammengesetzt konzeptualisiert wird. Andererseits, dass eine Entität als intern komplex konzeptualisiert wird, hat nicht notwendigerweise zur Folge, dass sie auch als partikularisiert betrachtet wird. Langacker (1991: 78) exemplifiziert diesen Sachverhalt mit den englischen Ausdrücken boards und lumber. Beide Ausdrücke konzeptualisieren zusammengesetzte Entitäten. Nur im Fall von boards sind jedoch auch die konstitutiven Bestandteile hervorgehoben.

Die Existenz eines konzeptuellen Subsystems der Multiplexität, das aus den vier oben beschriebenen Kategorien besteht, ist funktional begründet. Diese Kategorien werden in unterschiedlichen grammatikalischen Konstruktionen miteinander kombiniert, um die Beschaffenheit komplexer Eintitäten zu konzeptualisieren. Das heiß: die Konzeptualisierung komplexer Entitäten setzt voraus, dass wir diesen Kategorien einen bestimmten Wert zuweisen.

Insgesamt besteht das konzeptuelle System der Multiplexität aus den folgenden semantischen Kategorien und Werten:

20 Das, was Langacker profiling nennt. Vgl. u.a. Langacker (1999: 27ff). 


\begin{tabular}{l} 
Semantische Kategorien \\
\begin{tabular}{|l|l|}
\hline \multirow{2}{*}{ Plexity } & Werte \\
\cline { 2 - 2 } & Uniplex \\
\hline \multirow{2}{*}{ State of Boundedness } & Begrenzt \\
\cline { 2 - 2 } & Unbegrenzt \\
\hline \multirow{2}{*}{ State of Dividedness } & Zusammengesetzt \\
\cline { 2 - 2 } & Kontinuierlich \\
\hline Partikularisierung & Partikularisiert \\
\cline { 2 - 2 } & Nicht-partikularisiert \\
\hline
\end{tabular} \\
\hline
\end{tabular}

Tabelle 1: Kategorien und Werte des konzeptuellen Subsystems der Multiplexität

Abschließend ist noch zu beachten, dass neben der bereits angesprochenen Beziehung zwischen den Kategorien state of dividedness und Partikularisierung noch eine weitere wichtige Relation zwischen Kategorien bzw. Werten besteht. Wie Jackendoff (1990: 20-22) richtig beobachtet, ist der Wert multiplex (PLURAL, bei Jackendoff) im Grunde als zusammengesetzt aus den Werten unbegrenzt ("-bounded") und zusammengesetzt (bei Jackendoff: "+internal structure") analysierbar. Konsequenterweise besteht uniplex aus den entgegengesetzten Werten begrenzt und kontinuierlich.

Zieht man dies in Betracht, so kommt man zur folgenden (reduzierten) Konstellation von semantischen Werten für das Subsystem der Multiplexität:

$$
\begin{array}{rll}
{[\text { begrenzt }]} & \text { vs. } & {[\text { unbegrenzt }]} \\
{[\text { zusammengesetzt }]} & \text { vs. } & {[\text { kontinuierlich }]} \\
{[\text { partikularisiert }]} & \text { vs. } & {[\text { nicht-partikularisiert }]}
\end{array}
$$

\section{Die grammatikalische Konzeptualisierung der Multiplexität von Gegegenständen im GBP}

Wir sind nun in der Lage, das entwickelte Modell auf die Beschreibung des semantischen Pols der in der Einleitung vorgestellten grammatikalischen Konstruktionen des GBP anzuwenden. Dabei soll zunächst gezeigt werden, dass sich beide Konstruktionen darin unterscheiden, mit welchen Werten sie jeweils die drei Kategorien belegen. Anschließend werden wir uns etwas genauer der ersten Konstruktion widmen und dabei zeigen, dass sie je nach der Art des verwendeten Lexems in unterschiedlichen semantischen Subtypen untergliedert werden kann.

Alle verwendeten Beispiele stammen aus dem Corpus Gesprochenes Portugiesisch Brasiliens der Universität Bielefeld. Das Corpus besteht aus Transkriptionen von unterschiedlichen Gesprächen, die zwischen 1997 und 1999 in der Region Centro-Oeste Brasiliens aufgenommen wurden. ${ }^{21}$

\footnotetext{
${ }^{21}$ Das Corpus entstand im Rahmen des vom Land NRW und des DAAD finanzierten Forschungsprojekts "LaieExperte Interaktion". Das Projekt wurde von Prof. Dr. Reinhard Meyer-Hermann (Universität Bielefeld) 


\subsection{Grammatikalische Konstruktionen}

Wir wiederholen hier für praktische Zwecke die Abbildungen der beiden grammatikalischen Konstruktionen, die in der Einleitung bereits vorgestellt und formal charakterisiert wurden.

(a)

\section{[PL DET N]}

(b)

\section{[DET N]}

\section{Abbildung 2}

Betrachten wir zuerst NPs aus unserem Corpus, die nach der Konstruktion (a) gebildet werden:
(a) .... gente precisou
fazer
as
casinha...
wir brauchen-Prät.-1Pers.-pl.
machen
Art. def.-Pl.
Haus-Diminutiv
'wir mussten die kleinen Häuser bauen'
(b) $\quad$..eu
conversei
com as
menina...
mit Art. def.-pl. Mädchen

ich unterhalten-Prät.-1Pers.-sg.
'ich unterhielt mich mit den Mädchen'

(c)

$\begin{array}{llll}\text {...agora } & \text { os } & \text { companheiro } & \text { fala... } \\ \text { jetzt } & \text { Art. def.-pl. } & \text { Kumpel } & \text { sprechen-Präs.-3Pers.sg. }\end{array}$

'jetzt sprechen die Kumpel'

(d)

$\begin{array}{lllll}\text {...deixa } & \text { eu } & \text { ver } & \text { as } & \text { bezerra... } \\ \text { lassen-Imper.-2Pers.sg. } & \text { ich } & \text { sehen } & \text { Art. def.-pl. } & \text { Kälber-weib. }\end{array}$

'lass mir mal die Kälber sehen'

(e) ...aí

o senhor

compara

os

itens...

dann

Sie

vergleichen-präs.-3-pers.sg.

Art.-def.-pl.

Punkt-pl.

'dann vergleichen Sie die Punkte'

(f) $\quad \ldots$ os

médio

produtores...

Art.-def.-pl. mittelgroß

Erzeuger-pl.

'die mittelgroßen Erzeuger'

(g) $\quad$.. eu

estou calculando

essas

carreta...

ich

bin berechnen-gerund. diese-pl.

LKW-Ladung

'ich bin dabei, die LWK-Ladungen zu berechnen'

(h) $\quad$.. vou

tratar

desses

animais...

Fut.-1Pers.-sg.

behandeln

von-Demons.-pl.

Tier-pl.

'ich werde diese Tiere behandeln'

(i) ...porque que você acha

warum dass du glauben-Präs.-3Pers.sg.

que tem essas fábrica de ração aí...

dass es gibt Demons.-pl. Fabrik von Futter dort

'was glaubst du denn, warum es dort diese Futterfabriken gibt'

koordiniert. Die Aufnahmen wurden vorwiegend in unterschiedlichen Ortschaften des Bundesstaates Goiás gemacht. 


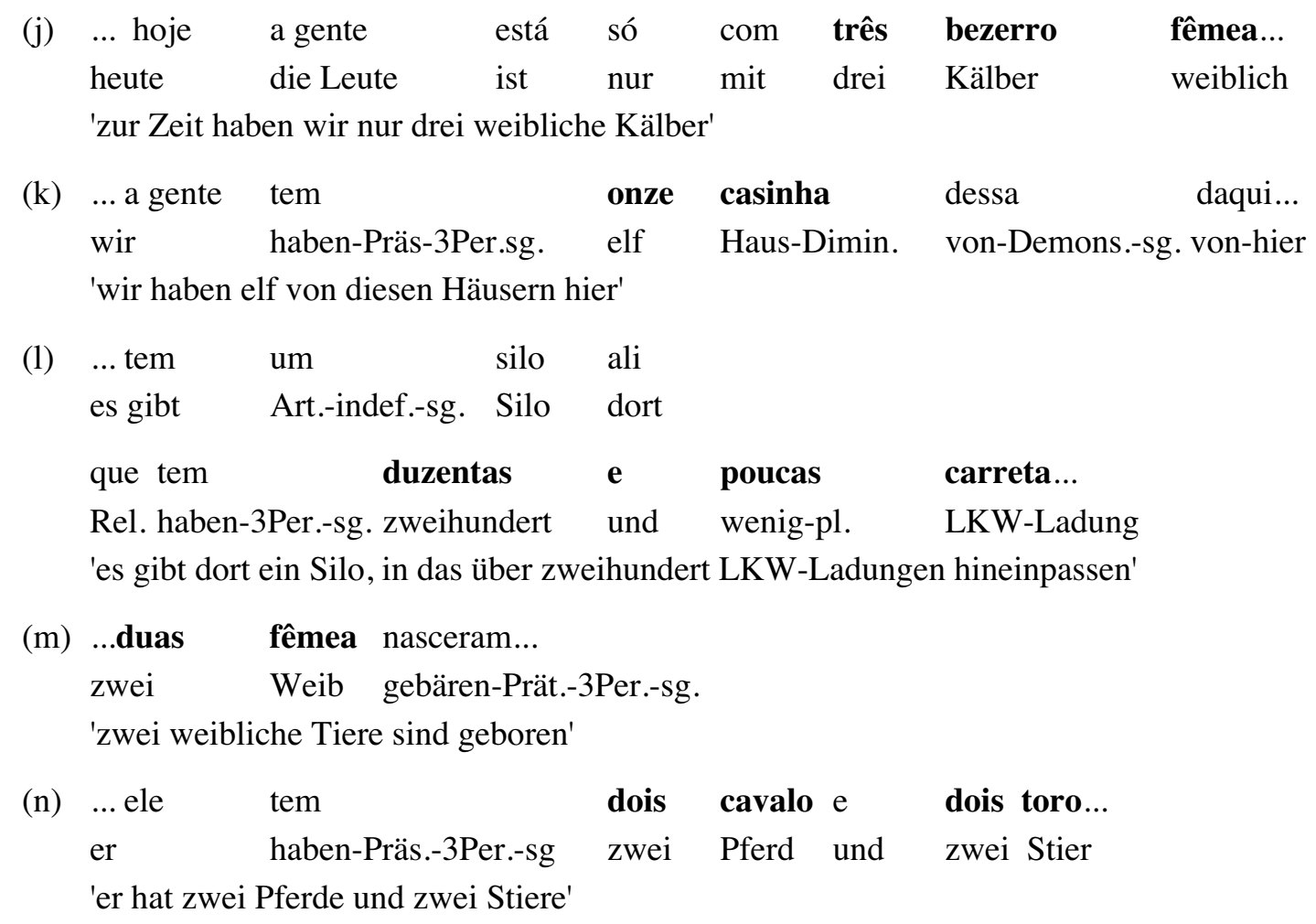

Formal zeichnen sich alle markierten NPs der Äußerungen in (4) dadurch aus, dass sie einen offen realisierten Pluralisierer haben. Sie entsprechen hiermit also dem zentralen differenzierenden formalen Merkmal der nominalen Konstruktion (a) (vgl. oben Abb. 2). In den meisten Fällen handelt es sich beim verwendeten Pluralisierer um das Suffix -s: (4)(a) as casinha, (4)(b) as menina, (4)(c) os companheiro, (4)(d) as bezerra, (4)(e) os itens, (4)(f) os médio produtores, (4)(g) essas carreta, (4)(h) esses animais, (4)(i) essas fábrica. In (4)(j) três bezerro fêmea, (4)(k) onze casinha, (4)(1) duzentas i poucas carreta, (4)(m) duas fêmea und (4)(n) dois cavalo e dois toro wird Pluralität dagegen lexikalisch markiert (in allen Fällen durch Quantifizierer). In nur drei Fällen wird Pluralität an mehr als einer Stelle markiert ((4)(e) os itens, (4)(f) os médio produtores und (4)(h) esses animais. Wenn Pluralität an nur einer Stelle markiert wird, handelt es sich dabei stets um die Determinierer-Position (DET). Wenn zwei Pluralisierer verwendet werden, werden sie jeweils am Determinierer (DET) und am Nomen $(\mathrm{N})$ realisiert. Die untersuchten Beispiele bestätigen hiermit - jedenfalls tendenziell - die bereits von einigen Autoren für das GBP aufgestellte Hypothese der selektiven Markierung von Numerus (vgl. dazu oben Kap. 0).

Semantisch charakterisieren sich alle markierten NPs der Äußerungen in (4) (also unabhängig von der Anzahl und der Positionierung der Pluralisierer) dadurch, dass sie jeweils eine Mehrzahl von Gegenständen bezeichnen. Zusätzlich drücken die markierten NPs in (4)(j) três bezerro fêmea, (4)(k) onze casinha, (4)(1) duzentas i poucas carreta, (4)(m) duas fêmea und (4)(n) dois cavalo e dois toro aufgrund der Verwendung von Quantifizierern jeweils genau quantifizierte Mengen aus. In den anderen Fällen bezeichnen die fett markierten NPs lediglich eine (nicht weiter quantifizierte) Mehrzahl von Gegenständen. Ungeachtet der weiteren semantischen Spezifizierung durch den jeweils verwendeten Quantifizierer ist allen Beispielen gemeinsam, dass sie auf eine Mehrzahl von Objekten referieren. Sie zeichnen sich 
also durch die - für Pluralreferenz konstitutiven (vgl. dazu oben Kap. 2) - semantischen Werte [unbegrenzt] und [zusammengesetzt $]$ aus.

Ferner ist für die Analyse der semantischen Struktur dieser Beispiele wichtig, dass es sich bei den Referenten von allen NPs um Dinge handelt, mit denen man normalerweise einzeln interagiert, d.h.: zu den 'interaktiven Eigenschaften'22 dieser Dinge gehört, dass sie im Alltag in der Regel so vorkommen, dass sie autonome Einheiten unserer Erfahrung ('Individuen' im allgemeinen semantischen Sinne) darstellen und dass wir uns in unseren täglichen Handlungen mit ihnen einzeln auseinandersetzen. Wir haben es in unseren alläglichen Handlungen typischerweise nicht mit großen Mengen an Fabriken ((4)(i)), LKWs ((4)(1)), Häusern $((4)(\mathrm{k}))$, etc. zu tun. Die Konzeptualisierung einer Mehrzahl solcher Dinge hebt diese interaktiven Eigenschaften nicht auf. Das heißt: die Konzeptualisierung der Mehrzahl resultiert aus der Replikation des diskreten, (interaktiv) autonomen Dings, das von dem jeweiligen Nomen im Singular bezeichnet wird. Konstitutiv für die Beispiele in (4) ist also ein hoher Grad an Partikularisierung. Bei den bezeichneten zusammengesetzten Entitäten werden die konstitutiven Bestandteile - also die einzelnen diskreten Dinge - hervorgehoben und nicht etwa der Einheitscharakter der Zusammensetzung als Ganzes.

In manchen Fällen werden die einzelnen konstitutiven Elemente durch die Verwendung von Numeralia (vgl. (4)(j), (k), (l), (m), und (n)) oder Demonstrativa (in deiktischer Lesart, vgl. (4)(g), (h) und (i)) zusätzlich partikularisiert. Es handelt sich dabei um eine zusätzliche Partikularisierung, bzw. um eine Inkrementierung der Partikularisierung insofern, als das ausdrückliche Hinweisen auf einzelne (!) Dinge mittels Demonstrativa sowie die genaue Quantifizierung unterschiedlicher (!) Elemente mittels Numeralia bereits das Vorhandensein partikularisierter Elemente vorausssetzt. ${ }^{23}$

Insgesamt zeichnen sich die NPs in (4) - was das konzeptuelle Subsystem der Multiplexität angeht - durch folgende Konstellation semantischer Werte aus:

\section{[[unbegrenzt $],[$ zusammengesetzt $],[$ partikularisiert $]]$}

Betrachten wir nun NPs aus unserem Corpus, die nach der Konstruktion (b) gebildet wurden:
(a) .... Acabou
o fósforo ...
Aufbrauchen/alle sein-3Pers.-sg.-Prät.
der Streichholz 'die Streichhölze sind alle'
(b) $\quad \ldots \mathrm{Eu}$ ich
vou
comprar 1Pers.-sg.-Fut. kaufen
'ich werde die Mangos kaufen'

\footnotetext{
${ }^{22}$ Das Konzept der "interaktiven Eigenschaften" geht auf Rosch (1978: 29ff) zurück und wurde später vor allem von Lakoff (1987: 50ff) weiterentwickelt.

${ }^{23}$ Es geht hier nicht um das simplistische Argument, dass Zählen Zählbarkeit voraussetzt, sondern vielmehr um den Sachverhalt, dass Zählen - oder genauer, das Erfassen durch Numeralia - die konzeptuelle Konstruktion von Zählbarkeit (durch linguistische Mittel) präsupponiert.
} 
(c) ... quanto custa a laranja? ... wieviel kostet die-sg. Orange 'wieviel kosten die Orangen?'

(d) ...ficou um pouco bleiben-Prät.-3Pers.-sg. Art.-indef.-sg. bißchen prejudicada por causa de uma cigarrinha beeinträchtigen-Part aufgrund Gen. Art.-indef.-sg. Zikade 'sie wurde etwas beeinträchtigt durch die Zikaden'

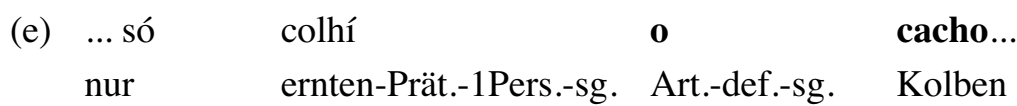
'ich habe nur die Kolben (der Hirse) geerntet'

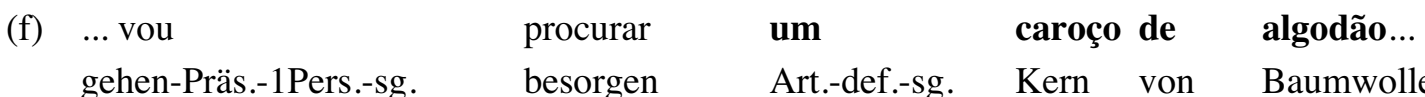
gehen-Präs.-1Pers.-sg. besorgen Art.-def.-sg. Kern von Baumwolle 'ich werde Baumwollkerne besorgen'

(g) ...você faz um trem du machen-Präs.-3Pers.-sg. $\quad$ Art.-indef.-sg. Ding já pronto para o rato... schon fertig für Art.-def.-sg. Rate 'du machst ein fertiges (einsatzfertiges) Mittel gegen Raten'

(h) $\quad$...ontem recebí menos pelo $\quad$ ovo... gestern bekommen-Prät.-1Pers.-sg. weniger für-Art.-def.-sg. Ei 'gestern bekam ich weniger für die Eier'

(i) ... Luana, você já escovou o dente? ... Luana du schon putzen-2Pers.-sg.-Prät. der Zahn 'Luana, hast du dir schon die Zähne geputzt?'

(j) ... Vai lavar a a a gehen-2Pers.-sg.-imper. waschen die-sg. Hand 'geh mal die Hände waschen!'

(k) ... O meu pai sempre deixa der mein Vater immer lassen-3Pers.-sg.-Präs.

a chuteira no portamalas ...

die-sg. Fussballschuh im Kofferraum

'mein Vater lässt immer die Fussballschuhe im Kofferraum'

(1) ... minha tia está fazendo

meine Tante sein-3Per-sg.-Präs. machen-Gerund.

a unha na Geralda...

Art.-def.-sg. Nagel in-Art.-def.-sg. Geralda

'meine Tante lässt sich gerade die Fingernägel bei Geralda lackieren' 
Formal zeichnen sich alle markierten NPs der Äußerungen in (5) dadurch aus, dass sie keinen offen realisierten Pluralisierer haben. Sie entsprechen hiermit also dem zentralen differenzierenden formalen Merkmal der nominalen Konstruktion (b) (vgl. oben Abb. 2).

Trotz dieses gemeinsamen formalen Merkmals können die markierten NPs in (5) nach semantischen Kriterien in zwei unterschiedliche Gruppen klassifiziert werden. Die NPs in den Beispielen (5)(a) bis (5)(h) zeichnen sich dadurch aus, dass sie - ungeachtet fehlender Pluralmarkierung - ganz klar auf eine Mehrzahl von Objekten referieren. (Um dies an nur einigen Beispielen erneut klar zu machen: Etwa in (5)(d) meint der Sprecher durch die Verwendung der (nicht-pluralmarkierten) NP uma cigarrinha natürlich nicht, dass die Ernte durch eine einzige Zikade beeinträchtigt wurde, sondern durch viele; Analog dazu verwendet der Sprecher der Äußerung in (5)(e) die (nicht-pluralmarkierte) NP o cacho nicht etwa, um zu behaupten, dass er einen einzigen Hirsekolben geerntet hat, usw.) Genauso wie die Beispiele unter (4) zeichnen sich auch diese NPs also durch die - für Pluralreferenz konstitutiven (vgl. dazu oben Kap. 2) - semantischen Werte [unbegrenzt] und [zusammengesetzt $]$ aus.

Für die Analyse der semantischen Struktur der Beispiele in (5)(a) bis (5)(h) ist jedoch auch wichtig, dass es sich bei den Referenten von allen markierten NPs um Dinge handelt, mit denen man normalerweise nicht einzeln interagiert: ${ }^{24}$ Man verkauft zum Beispiel in der Regel nicht nur ein Ei, sondern mehrere (5)(h); man erntet normalerweise nicht nur einen Hirsekolben, sondern viele (5)(e); man besorgt nicht nur einen Baumwollkern, usw. Zu den interaktiven Eigenschaften dieser Dinge gehört, dass wir ihnen in der Regel im Alltag bereits in der Mehrzahl begegnen und insofern auch sie in der Mehrzahl konzeptualisieren. Einzelne von ihnen kommen relativ selten als autonome Einheiten unserer Erfahrung vor. Und wir setzen uns auch eher selten mit einzelnen von ihnen in unseren täglichen Handlungen (verkaufen, ernten, etc.) auseinander.

Wenn einzelne Vorkommnise eines bestimmten Typs von Ding keine normale, bzw. prototypische Form unserer Erfahrung darstellen, ist es nahe liegend, dass die entsprechende Mehrzahl nicht als eine Gruppe (interaktiv) autonomer, diskreter Einheiten konzeptualisiert wird. Anders als bei den NPs in (4) gehört hier zur Konzeptualisierung der Mehrzahl ein relativ niedriger Partikularisierungsgrad. Die Referenten der NPs (5)(a) bis (5)(h) charakterisieren sich also durch das semantische Merkmal [nicht-partikularisiert].

Insgesamt haben wir für die Referenten der fett markierten NPs in (5)(a) bis (5)(h) folgende Konstellation von semantischen Merkmalen:

\section{[[unbegrenzt $],[$ zusammengesetzt $],[$ nicht-partikularisiert $]]$}

Ein ganz anderes Bild ensteht aus der Analyse der semantischen Struktur der markierten NPs in (5)(i) bis (5)(1). Die Referenten dieser NPs weisen zum Teil die gleichen semantischen

\footnotetext{
${ }^{24}$ Einige Autoren (vgl. etwa Behrens 1999: 58-62) haben richtig beobachtet, dass diese und andere interaktiven Eigenschaften stets kontextabhängig sind: dasselbe Referenzobjekt kann in unterschiedlichen Kontexten unterschiedliche interaktive Eigenschaften aufweisen und wird infolgedessen auch unterschiedlich konzeptualisiert. Nichtsdestoweniger kann man auf Grund von Frequenzkriterien wohl von (proto)typischen interaktiven Eigenschaften sprechen.
} 
Merkmale auf, die für (lexikalische) Kollektiva konstitutiv sind. Denn auf der einen Seite verfügen diese Referenten zwar über eine klare zusammengesetzte interne Struktur. Auf der anderen Seite werden sie jedoch trotz zusammengesetzter interner Struktur als begrenzte Entitäten konzeptualisiert. Genauso wie zum Beispiel das lexikalische Kollektivum Kommitee auf verschiedene Elemente (einzelne Mitglieder) referiert, die dennoch zusammen eine begrenzte Einheit bilden, so referiert etwa auch dente auf unterschiedliche Elemente, die dennoch zusammen eine einheitliche, begrenzte Struktur bilden.

Ein wichtiges Indiz für das Zutreffen dieser Lesart liefert uns das in vielen Fällen beobachtete Verhalten von Numeralia und anderen Quantifizierern (wie z. B. vários, numerosos etc.), die sich durch die semantische Eigenschaft [+ individualisiert] auszeichnen. Quantifizierer dieses Typs beziehen sich in den analysierten Fällen nicht (mehr) ${ }^{25}$ auf einzelne diskrete Entitäten, sondern auf die jeweilige Mehrzahl als einheitliches, begrenztes Ganzes:

(a) ...Ela já fez quatro unha hoje... sie schon machen-Prät.-3Pers.-sg. vier Nagel heute 'sie hat heute bereits vier Frauen die Fingernägel lackiert'

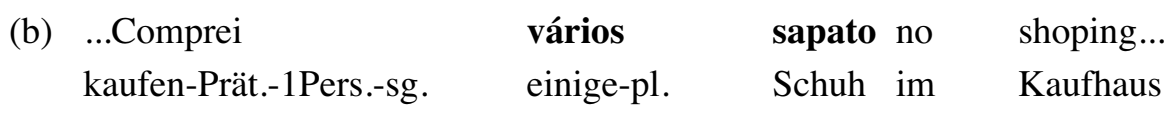
'ich habe einige Paar Schuhe im Kaufhaus gekauft'

In (6)(a) bezieht sich die Quantifikation durch das Numerale quatro nicht auf einen Fingernagel, sondern auf eine (begrenzte) Einheit bestehend aus allen zehn. Genauso bezieht sich der Quantifizierer vários in (6)(b) nicht auf einen einzelnen Schuh, sondern auf ein Paar Schuhe als Einheit. Bekanntlich besteht die Bedingung der Möglichkeit für die Quantifikation durch Quantifizierer dieses Typs (bzw. für die Pluralisierung im Allgemeinen) im Vorhandensein von begrenzten Referenzobjekten (vgl. dazu etwa Jackendoff 1990: 20-22). ${ }^{26}$ Die Referenten der NPs in (6) und in (5)(i) bis (5)(1) erfüllen offenbar diese Bedingung.

Die Konzeptualisierung solcher Referenten als begrenzte Einheiten trotz zusammengesetzter interner Struktur ist wahrscheinlich durch unterschiedliche Faktoren motiviert. Man kann (mindestens) drei solche Faktoren differenzieren: materielle Kontinuität, räumliche Kontiguität und funktionale Zusammengehörigkeit. Diese Faktoren können in einzelnen Fällen (in unterschiedlichen Graden) miteinander interagieren.

Objekte wie TESOURA (Schere), ALICATE (Zange), ÓCULO (Brille), BINÓCULO (Fernglas), CALÇA (Hose), PINÇA (Pinzette), ALGEMA (Handschelle), etc., die aus zwei symmetrischen Teilen bestehen, werden primär aufgrund materieller Kontinuität als begrenzte Einheit erfahren und konzeptualisiert. Die jeweiligen symmetrischen Teile sind miteinander so verbunden, dass sie nicht als diskrete Entitäten, sondern eher als Bestandteile eines einzigen (extern begrenzten) Dings betrachtet werden. Funktionale Motiviertheit spielt jedoch

\footnotetext{
${ }^{25}$ Mit Sicherheit handelt es sich um einen zwar nicht abgeschlossenen, aber bereits sehr fortgeschrittenen Entwicklungsprozess.

${ }^{26}$ Selbstverständlich können auch nicht-begrenzte Referenzobjekte pluralisiert werden. Dann enstehen aber bekanntlich andere Lesarten: der Wein - die Weine.
} 
auch eine wichtige Rolle. Denn die typischen Funktionen, für die wir solche Dinge in unseren täglichen Handlungen gebrauchen, sind nur durch die gemeinsame Verwendung beider konstitutiven Teile exekutierbar.

Bei Objekten wie SAPATO (ein Paar Schuhe), BOTA (ein Paar Stiefel), CHUTEIRA (ein Paar Fussballschuhe), LUVA (ein Paar Handschuhe), BRINCO (ein Paar Ohrringe), etc. besteht dagegen keine materielle Kontinuität zwischen den einzelnen Teilen. Sie werden vielmehr in erster Linie aufgrund räumlicher Kontiguität und sekundär auch wegen des funktionalen Zusammenhangs zwischen den bezeichneten Bestandteilen als (extern begrenzte) Einheit erfahren und konzeptualisiert.

Schließlich spielen Räumliche Kontiguität und funktionale Zusammengehörigkeit auch die Hauptrolle bei der Konzeptualisierung von Dingen wie zum Beispiel DENTE (Zähne) und UNHA (Fingernägel). Zur Konzeptualisierung dieser Elemente als begrenzte Einheit trägt allerdings auch das Vorhandensein einer übergeordneten Struktur bei (Gebiss und Hand).

Übrigens ist diese Form der Konzeptualisierung und ihre Kodierung durch nichtpluralmarkierte NPs weder eine Besonderheit des GBP noch des Portugiesischen im Allgemeinen. Man vergleiche etwa dt. Brille vs. engl. spectacles oder sp. gafas. Im GBP ist diese Form der Kodierung im Vergleich zum schriftsprachlichen brasilianischen Standard allerdings als häufiger einzustufen. Im schriftsprachlichen brasilianischen Standard werden durch die nicht-pluralmarkierte Form vor allem Objekte kodiert, die aus zwei symmetrischen Teilen bestehen, wie z. B. TESOURA (Schere), ALICATE (Zange), CALÇA (Hose), PINÇA (Pinzette), ALGEMA (Handschelle) etc. (vgl. Novo Aurélio 1999). Es gibt jedoch durchaus Ausnahmen, wie zum Beispiel ÓCULOS (Brille). In früheren Entwicklungsphasen der Sprache scheint dagegen der Plural auch für diese Fälle die standardisierte schriftsprachliche Form gewesen zu sein (vgl. u. a. dazu Nunes 1945: 232). Die Kodierung aller anderen besprochenen Fälle durch die nicht-pluralmarkierte Form ist dagegen als eine Besonderheit des GBP einzustufen.

Zur semantischen Struktur der Referenten der NPs in (5)(i) bis (5)(1) gehört schließlich auch das semantische Merkmal [nicht-partukularisiert]. Im Vordergrund steht hier der Einheitscharakter des jeweiligen Referenten, während der Umstand, dass diese Referenten intern komplex sind, also aus prinzipiell diskriminierbaren Bestandteilen bestehen, in der Konzeptualisierung im Hintergrund bleibt. Trotz der zusammengesetzten internen Struktur von Objekten wie zum Beispiel TESOURA, ÓCULO etc. wird bei ihrer Konzeptualisierung nicht zuletzt aus funktionalen Gründen - hervorgehoben, dass es sich jeweils um ein Objekt handelt.

Insgesamt kommen wir zur folgenden Konstellation von semantischen Merkmalen für die Referenten der NPs in (5)(i) bis (5)(1):

[[begrenzt], [zusammengesetzt $]$, [nicht-partikularisiert $]]$ 


\section{$5 \quad$ Schlussfolgerung}

Die folgende Tabelle fasst die bisherigen Ergebnisse der vorliegenden Untersuchung zusammen.

\begin{tabular}{|c|c|c|}
\hline $\begin{array}{l}\text { Grammatikalische } \\
\text { Konstruktion }\end{array}$ & Beispiel & Semantische Merkmale \\
\hline [PL DET N] & As laranja(s) (die Orangen) & [unbegrenzt, zusammengesetzt, partikularisiert] \\
\hline \multirow{4}{*}{ DET N] } & A laranja (die Orange) & [begrenzt, kontinuierlich, nicht-partikularisiert] \\
\hline & O óculo (die Brille) & [begrenzt, zusammengesetzt, nicht-partikularisiert] \\
\hline & A farinha (das Mehl) & [unbegrenzt, kontinuierlich, nicht-partikularisiert] \\
\hline & A laranja (die Orangen) & [unbegrenzt, zusammengesetzt, nicht partikularisiert] \\
\hline
\end{tabular}

Tabelle 2: grammatikalische Konstruktionen mit jeweiligen Bündeln semantischer Merkmale

Die in der Tabelle dargestellten Bündel semantischer Merkmale konstituieren jeweils unterschiedliche semantische Pole der grammatikalischen Konstruktionen. ${ }^{27}$ Neben den im Kapitel 3 bereits besprochenen Bündeln semantischer Merkmale umfasst die Tabelle zwecks Vergleichs zwei zusätzliche (durch grauen Hintergrund markierte) Bündel, die weitere typische Referenzobjekte der nicht-pluralisierten Konstruktion charakterisieren. Es handelt sich dabei jeweils um die konstitutiven semantischen Merkmale für (zählbare) Entitäten (z. B. LARANJA) und jene, die typischerweise Massen (z. B. FARINHA) zugrunde liegen.

Bei der Analyse dieser Tabelle sind einige zentrale Punkte besonders hervorzuheben. Zunächst ist bemerkenswert, dass das gleiche Lexem in unterschiedlichen Konstruktionen mit unterschiedlichen Bündeln von semantischen Werten fungieren kann. Das Wort laranja kann beispielsweise in der pluralmarkierten NP as laranja(s) eine unbegrenzte, zusammengesetzte Gruppe partikularisierter Entitäten und in der nicht-pluralmarkierten NP a laranja entweder eine begrenzte, kontinuierliche und nicht-partikularisierte Entität (also das, was man traditionell ein zählbares Ding nennt) oder aber eine unbegrenzte, zusammengesetzte, aber nichtpartikularisierte Menge von Entitäten bezeichnen. D.h.: die konstatierten semantischen Unterschiede sind nicht auf der lexikalischen, sondern auf der NP-Ebene zu platzieren. Es handelt sich also um den semantischen Beitrag der jeweiligen nominalen Konstruktion, um das also, was wir den semantischen Pol der Konstruktion genannt haben. Das scheint die von einigen Autoren bereits aufgestellte Hypothese zu bestätigen, dass portugiesische Lexeme nicht über die semantischen Merkmale verfügen, die die Eigenschaft 'Zählbarkeit' ausmachen. Diese Eigenschaft existiert erst auf der NP-Ebene (vgl. Blühdorn/Simões/Schmaltz 2004: 35). Ungeachtet der Richtigkeit dieser allgemeinen Beobachtung kann man jedoch nicht außer Acht lassen, dass es durchaus klare kontextuelle Restriktionen gibt, die gewisse Kombinationen von bestimmten Lexemen mit einer der besprochenen grammatikalischen Konstruktionen bzw. mit der von der Konstruktion festgelegten Lesart ausschließen. Es ist zum Beispiel recht schwer, sich eine Verwendungssituation vorzustellen, wo die nicht-

\footnotetext{
${ }^{27}$ Die Referenzmöglichkeiten dieser Konstruktionen schöpfen sich natürlich hiermit nicht aus.
} 
pluralmarkierte NP a casa auf eine unbegrenzte, zusammengesetzte und nicht-partikularisierte Menge von Entitäten referieren kann. ${ }^{28}$

Diese letzte Beobachtung führt uns zu einem zweiten wichtigen Punkt. Eine NP, die nach der nicht-pluralmarkierten Konstruktion gebildet wird (z. B. a laranja), ist prinzipiell ambig. Sie kann entweder auf ein Einzelding (die Orange) oder auf eine (unbegrenzte, zusammengesetzte und nicht-partikularisierte) Menge von Dingen (die Orangen) referieren. In solchen Fällen ist der Kontext der zentrale Desambiguierungsfaktor. Die konkrete Verwendungssituation wird klar machen, ob sich die NP auf ein bestimmtes, einzelnes Ding ('die Orange auf dem Tisch') oder aber auf eine Menge von Dingen ('die Orangen im Supermarktlager') bezieht.

Vergleicht man alle Bündel semantischer Merkmale miteinander, so ist ferner festzustellen, dass die Kategorie Partikularisierung das zentrale bedeutungsdifferenzierende Element zwischen den beiden Konstruktionen darstellt. Das semantische Merkmal [partikularisiert] wird nur von der pluralmarkierten Konstruktion kodiert. Die nicht-pluralmarkierte Konstruktion ist dagegen für dieses Merkmal nicht sensitiv. Sie kodiert entweder intern nicht zusammengesetzte (kontinuierliche) und insofern auch nicht-partikularisierbare Entitäten (Maße oder zählbare Einzeldinge) oder zusammengesetzte, aber nicht-partikularisierte Entitäten (begrenzte, zusammengesetzte und nicht-partikularisierte Entitäten wie O ÓCULO und unbegrenzte, zusammengesetzte und nicht-partikularisierte Entitäten wie A LARANJA). Hierdurch entsteht eine besondere semantische und funktionale Komplementarität zwischen den beiden Konstruktionen bei der Kodierung der Mehrzahl von Objekten. Für die Referenz auf partikularisierte Mengen von Objekten verwendet man im GBP plural-markierte NPs, während sich nicht-pluralmarkierte NPs auf nicht-partikularisierte Mengen von Objekten beziehen.

Betrachten wir nun etwas genauer die in der Tabelle 2 angegebenen Referenzmöglichkeiten der nicht-pluralmarkierten Konstruktion. Das GBP kodiert mittels nicht-pluralmarkierter NPs sowohl kontinuierliche (CASA) als auch zusammengesetzte begrenzte Entitäten (ÓCULO). Die zweitgenannte Referenzmöglichkeit existiert zwar auch in einigen Fällen im schriftsprachlichen brasilianischen Portugiesichen, ist aber bei weitem nicht so breit verbreitet. Analog dazu kodiert das GBP mittels nicht-pluralmarkierter NPs sowohl unbegrenzte und kontinuierliche Entitäten (also typische Massen, wie etwa FARINHA) als auch unbegrenzte, aber zusammengesetzte Entitäten (wie etwa LARANJA, FEIJÃO etc.). Diese letzte Referenzmöglichkeit existiert nicht im schriftsprachlichen brasilianischen Portugiesichen. Wenn wir unterstellen, dass die in beiden Fällen jeweils letztgenannten Referenzmöglichkeiten von nicht-pluralmarkierten NPs auch nicht in früheren Entwicklungsstadien des GBP existierten, haben wir es wohl mit einem Erweiterungsprozess des Referenzspektrums dieser Konstruktion zu tun, der als Ausgangspunkt die für nicht-pluralmarkierte NPs typischen Referenzobjekttypen 'begrenztes, kontinuierliches Ding' und 'unbegrenzte, kontinuierliche Masse' hat.

\footnotetext{
28 Wie oben bereits suggeriert wurde, handelt es sich konkret um die interaktiven Eigenschaften, die ein bestimmtes Ding in einem bestimmten Kontext aufweist. Diese Relativierung schließt prinzipiell nicht aus, dass ein Kontext gefunden werden kann, wo die NP a casa in der Tat auf eine solche Menge referieren kann.
} 
Abschließend noch eine Bemerkung allgemeinerer Natur: die vorliegende Analyse bestätigt die anderenorts bereits realisierte Beobachtung, dass sich semantische Kategorien wie etwa zählbar versus nicht-zählbar (Masse), Singular vs. Plural, etc. für die Beschreibung der Kodierungsleistungen von einigen (wahrscheinlich sogar von vielen) Sprachen als zu grobgranular erweisen (vgl. u. a. Behrens 1995, Behrens/Sasse 1999 und Blühdorn/Simões/Schmalz 2004). Um die konzeptuellen Distinktionen zwischen den beiden untersuchten Konstruktionen des GBP adäquat zu erfassen, war es nötig, von weiter dekomponierten Kategorien auszugehen.

\section{Literaturangaben}

Askedal, John Ole (2001): "Conceptions of typological change". In: Haspelmath, Martin/ König, Ekkehard/Oesterreicher, Wulf/Raible, Wolfgang (eds.): Language Typology and Language Universals. An International Handbook. 2. Halbband. Berlin: 1624-1640.

Behrens, Leila (1995): "Categorizing between lexicon and grammar. The MASS/COUNT distinction in a cross-linguistic perspective". Lexicology 1: 1-112.

Behrens, Leila/Sasse, Hans-Jürgen (1999): Qualities, Objetcs, Sorts, and Other Treasures: GOLD-digging in English and Arabic. Köln.

Blühdorn, Hardarik/Simões, Luciene/Schmalz, Márcia (2004): Sintagmas Nominais contáveis e não-contáveis no alemão e no português brasileiro. (Manuskript).

Clausner, Timothy C./Croft, William (1999): "Domains and image schemas". Cognitive Linguistics 10-1: 1-31.

Corbett, Grewille G. (2000): Number. Cambridge.

Croft, William (1999): "Some contributions of typology to cognitive linguistics, and vice versa". In: Jansen, Theo/Redecker, Gisela (eds.): Cognitive Linguistics: Foundations, Scope, and Methodology. Berlin/New York: 61-93.

Croft, William (2002): Radical Construction Grammar. Syntactic Theory in Typological Perspective. Oxford.

Croft, William (2004): "Logical and typological arguments for Radical Construction Grammar". Manuskript. Erscheint in: Fried, Mirjam/Östman, Jan-Ola (eds.): Construction Grammar(s): Cognitive and cross-language dimensions. Philadelphia.

Farrell, Patrick (1998): "The Conceptual Basis of Number Marking in Brazilian Portuguese". In: Koenig, Jean-Pierre (ed.): Discourse and Cognition. Bridging the Gap. Standford: 3-16.

Gärtner, Eberhard (1996): "Substandard-Phänomene im brasilianischen und afrikanischen Portugiesisch". Neue Romania 17: 245-276.

Große, Sybille/Zimmermann, Klaus (eds.) (1998): Substandard e mudança no português do Brasil. Frankfurt am Main.

Jackedoff, Ray (1991): "Parts and boundaries". Cognition 41: 9-45.

Jackedoff, Ray (2002): Foundations of Language. Brain, Meaning, Grammar, Evolution. Oxford.

Lakoff, George (1987): Women, Fire, and Dangerous Things. What Categories Reveal about the Mind. Chicago/London. 
Langacker, Ronald W. (1987): Foundations of Cognitive Grammar. Volume 1: Theoretical Prerequisites. Standford.

Langacker, Ronald W. (1991a): Foundations of Cognitive Grammar. Volume 2: Descriptive Applications. Standford.

Langacker, Ronald W. (1991b): "Cognitive Grammar". In: Droste, Flip G./Joseph, John E. (eds.): Linguistic Theory and Grammatical Description. Amsterdam/Philadelphia: 275306.

Langacker, Ronald W. (1999): "Assessing the cognitive linguistic enterprise". In: Jansen, Theo/Redecker, Gisela (eds.): Cognitive Linguistics: Foundations, Scope, and Methodology. Berlin/New York: 13-59.

Naro, Anthony Julius/Pereira Scherre, Maria Marta (2000): "Variable Concord in Portuguese: The Situation in Brazil and Portugal". In: Mcwhorter, J. (ed.): Language Change and Language Contact in Pidgins and Creoles. Amsterdam/Philadelphia: 235-255.

Nunes, José Joaquim (1945): Compêndio de gramática histórica portuguesa: Fonética e Morfologia. Lisboa.

Pereira Scherre, Maria Marta (1994): "Aspectos da concordância de número no português do Brasil". Revista Internacional de Língua Portuguesa 12: 37-49.

Pereira Scherre, Maria Marta (1998): "Variação da concordância nominal no português do Brasil: influência das variáveis posição, classe gramatical e marcas precedentes". In: Große, Sybille/Zimmermann, Klaus (eds.): 153-188.

Pereira Scherre, Maria Marta/Naro, Anthony Julius (1997): "A concordância de número no português do Brasil: um caso típico de variação inerente". In: Oliveira, Dermal da Hora (ed.): Diversidade lingüística no Brasil. João Pessoa: 3-114.

Roberts, Ian/Kato, Mary A. (eds.) (1996): Português Brasileiro. Uma viagem diacrônica. Campinas.

Ronneberger-Sibold, Elke (1997): "Typology and the diachronic evolution of German morphosyntax". In: Fisiack, Jacek (ed.): Linguistic Reconstruction and Typology. Berlin/New York: 313-335.

Rosch, Eleanor (1978): "Principles of Categorization". In: Rosch, Eleanor/Lloyd B. B. (eds.): Cognition and Categorization. Hillsdale: 27-48.

Talmy, Leonard (2002): Toward a Cognitive Semantics. Volume I: Concept Structuring Systems. Cambridge, MA (MIT Press).

Vazzata-Dias, Juçá Fialho (2000): "A Concordância de Número nos Predicativos/Participios Passivos na Fala do Sul do Brasil - Motivações extralingüísticas". Letras de Hoje 35, 1: 209-228. 